Proceedings

\title{
Promoting Reminiscences with Virtual Reality: Feasibility Study with People with Dementia ${ }^{+}$
}

\author{
Tiago Coelho *, Cátia Marques, Daniela Moreira, Maria Soares, Paula Portugal \\ and António Marques
}

School of Health - Polytechnic of Porto, Center for Rehabilitation Research (Psychosocial Rehabilitation Lab), 4200-072 Porto, Portugal

* Correspondence: tfc@ess.ipp.pt; Tel.: +351-222-061-000

+ Presented at the 2nd XoveTIC Conference, A Coruña, Spain, 5-6 September 2019.

Published: 26 July 2019

\begin{abstract}
This study aimed to examine the feasibility of promoting reminiscences with people with dementia, using $360^{\circ}$ videos presented with virtual reality headsets. Four individual sessions were conducted with nine people with dementia. Average duration of the exposure was approximately ten minutes. The experience appeared to be pleasant for most of the participants (with the exception of one of the participants who reported visual limitations). Most participants shared positive memories while viewing the recordings. None of the participants experienced any significant increase in symptoms associated with nausea and disorientation.
\end{abstract}

Keywords: dementia; reminiscence; virtual reality

\section{Introduction}

Dementia is a syndrome characterized by cognitive decline (affecting domains such as memory, language, attention and judgment), psychological and behavioural symptoms (such as depression, anxiety, apathy and aggression), and disability in everyday activities [1]. The prevalence of dementia is increasing worldwide, escalating caregiver burden and societal costs with social and health care [1]. Non-pharmacological treatments are essential to improve the well-being of people with dementia and their families. Some of these interventions seek to use preserved remote autobiographical memories to improve the patients' mood, behaviour and overall quality of life [2]. Reminiscence therapy is a well-supported example of these approaches, involving the discussion of past personal experiences, usually with the aid of prompts such as photos or objects [2]. However, there is a lack of evidence regarding the use of more immersive cues, such as permitted by virtual reality (VR). Therefore, this study aimed to assess the feasibility of promoting reminiscences with people with dementia, using $360^{\circ}$ videos of locations or activities relevant to each individual (considering their life story), displayed in VR headsets.

\section{Materials and Methods}

A convenience sample of 9 people with mild or moderate dementia, without severe visual deficits, was recruited. Participants had to have family members or other relevant individuals that could provide information about the participants' life story. Semi-structured interviews were conducted with patients and family members/informants to identify meaningful activities, contexts and events of the participants' past. Researchers analysed the content of the interviews and discussed which positive and pleasant memories could be depicted trough video recordings in $360^{\circ}$. A tailored program of four video recordings was defined for each individual, including settings such as childhood house and workplace locations, as well as leisure and religious venues. Reminiscence intervention consisted of four individual sessions conducted in two weeks. Each session included an 
initial preparation, exposure to one of the $360^{\circ}$ videos with a VR headset $(10-15 \mathrm{~min})$ and a final discussion. Questions regarding positive memories (associated with the video) were made during the exposure to promote their recollection and discussion. To assess the feasibility of this approach, the Simulator Sickness Questionnaire [3] was used, and an observation scale was prepared to register the participants' behaviour during the exposure.

\section{Results}

A preliminary analysis of the data showed that the average duration of the exposure was approximately $10 \mathrm{~min}$. The experience appeared to be pleasant for most of the participants, as they freely explored the $360^{\circ}$ environment. Participants, when encouraged or spontaneously, described what they were seeing or recalling during the exposure. Shared memories were mostly positive. Only one participant did not appear to enjoy the experience, showing some signs of agitation and asking to remove the headset after 5-6 min. This participant reported difficulty in seeing and understanding the environments and refused to participate in one of the sessions. Regarding simulator sickness, none of the participants experienced any significant increase in symptoms associated with nausea and disorientation. Mild eyestrain and blurred vision were reported in some cases.

\section{Discussion}

As no significant detriment to the well-being of the participants was observed, these findings demonstrate the feasibility of these kind of approaches. Future studies should focus on measuring the medium and long-term effects of promoting reminiscences with VR headsets, particularly on the psychological and behavioural symptoms of people with dementia.

Author Contributions: Conceptualization-T.C.; methodology-T.C., P.P.; formal analysis-T.C., C.M., D.M., M.S.; investigation-T.C., C.M., D.M., M.S.; resources-A.M.; preparing the original draft-T.C.; review and editing-T.C., P.P., A.M.; visualization-T.C.; project administration-T.C., P.P., A.M.

Funding: This research received no external funding.

Acknowledgments: The authors would like to thank the collaborating institutions that ensured the transportation of the participants and provided a location to perform part of the tasks of the research.

Conflicts of Interest: The authors declare no conflict of interest.

\section{References}

1. World Health Organization. Dementia: A Public Health Priority Geneva; World Health Organization: Geneva, Switzerland, 2012.

2. Woods, B.; O'Philbin, L.; Farrell, E.M.; Spector, A.E.; Orrell, M. Reminiscence therapy for dementia. Cochrane Database Syst. Rev. 2018, 3, Cd001120.

3. Kennedy, R.S.; Lane, N.E.; Berbaum, K.S.; Lilienthal, M.G. Simulator Sickness Questionnaire: An Enhanced Method for Quantifying Simulator Sickness. Int. J. Aviat. Psychol. 1993, 3, 203-220.

(C) 2019 by the authors. Licensee MDPI, Basel, Switzerland. This article is an open access article distributed under the terms and conditions of the Creative Commons Attribution (CC BY) license (http://creativecommons.org/licenses/by/4.0/). 